ARTICLE

DOI: $10.1038 / s 41467-017-02434-y$

\title{
Fibre-optic metadevice for all-optical signal modulation based on coherent absorption
}

\author{
Angelos Xomalis (1) 1,2, losif Demirtzioglou' ${ }^{1}$, Eric Plum (10 1,2, Yongmin Jung ${ }^{1}$, Venkatram Nalla (1) ${ }^{3}$ \\ Cosimo Lacava1, Kevin F. MacDonald ${ }^{1,2}$, Periklis Petropoulos (10 ${ }^{1}$, David J. Richardson ${ }^{1}$ \& Nikolay I. Zheludev ${ }^{1,2,3}$
}

Recently, coherent control of the optical response of thin films in standing waves has attracted considerable attention, ranging from applications in excitation-selective spectroscopy and nonlinear optics to all-optical image processing. Here, we show that integration of metamaterial and optical fibre technologies allows the use of coherently controlled absorption in a fully fiberized and packaged switching metadevice. With this metadevice, which controls light with light in a nanoscale plasmonic metamaterial film on an optical fibre tip, we provide proof-of-principle demonstrations of logical functions XOR, NOT and AND that are performed within a coherent fibre network at wavelengths between 1530 and $1565 \mathrm{~nm}$. The metadevice has been tested at up to 40 gigabits per second and sub-milliwatt power levels. Since coherent absorption can operate at the single-photon level and with $100 \mathrm{THz}$ bandwidth, we argue that the demonstrated all-optical switch concept has potential applications in coherent and quantum information networks.

\footnotetext{
${ }^{1}$ Optoelectronics Research Centre, University of Southampton, Southampton SO17 1BJ, UK. ${ }^{2}$ Centre for Photonic Metamaterials, University of Southampton Southampton SO17 1BJ, UK. ${ }^{3}$ Centre for Disruptive Photonic Technologies, School of Physical and Mathematical Sciences and The Photonics Institute, Nanyang Technological University, Singapore 637371, Singapore. Correspondence and requests for materials should be addressed to A.X. (email: ax1c15@soton.ac.uk) or to E.P. (email: erp@orc.soton.ac.uk) or to N.I.Z. (email: niz@orc.soton.ac.uk)
} 
A ll-optical signal processing fundamentally relies on modulation of one optical signal with another. Therefore alloptical logical functions have long been perceived as the exclusive domain of nonlinear optics ${ }^{1}$, which requires a minimum level of intensity to activate the nonlinear material response and faces trade-offs between magnitude and speed of the nonlinearity involved ${ }^{2-6}$. However, recently it was shown that an effective nonlinear response may be derived from coherent interaction of light with light on linear materials of substantially sub-wavelength thickness ${ }^{7}$. In contrast to conventional optical nonlinearities, the effect has been shown to allow intensity-independent control over absorption of light, from almost $0 \%$ to almost $100 \%{ }^{8}$, with 100 $\mathrm{THz}$ bandwidth ${ }^{9,10}$ and even for single-photon signals ${ }^{11}$. The concept has enabled all-optical control of luminescence ${ }^{12,13}$, redirection of light ${ }^{14,15}$ as well as control of nonlinear ${ }^{16}$, polarization ${ }^{17}$ and quantum ${ }^{18,19}$ effects in films of nanoscale thickness and excitation-selective spectroscopy ${ }^{20}$. In particular, it has been predicted that coherent interaction of light waves on lossy ultrathin films could perform signal processing functions ${ }^{7}$ and proof-of-concept experiments in the static regime have been reported $^{21,22}$.

Here, we report the realization of a switching network using a fully packaged, fiberized metamaterial device based on this concept. It uses a plasmonic nanostructure of substantially subwavelength thickness as a switchable absorber that allows the absorption of one optical pulse to be controlled by another coherent optical pulse. We demonstrate nonlinear input-output characteristics and all-optical operations analogous to logical functions NOT, AND and XOR at both $\mathrm{kHz}$ and $\mathrm{GHz}$ bitrates, in a fiberized configuration assembled from standard telecoms components, at wavelengths between 1530 and $1565 \mathrm{~nm}$.

\section{Results}

Coherent metamaterial absorption in a fibre network. As illustrated in Fig. 1a, the functionality of the network is based on controlling absorption of light with light on an ultrathin metamaterial absorber. It has two bidirectional ports, i.e. two inputs, $\alpha$ and $\beta$, and two outputs, $\gamma$ and $\delta$. The input waves propagate in opposite directions and, provided that they are mutually coherent, co-polarized and of equal intensity, they will form a standing wave with electric field nodes and anti-nodes (Fig. 1b). A sufficiently thin film may thus be placed at a node, where the electric field is zero due to destructive interference, or at an anti-node where the electric field amplitude is enhanced by constructive interference. Since truly planar structures interact with normally incident waves only via the electric field ${ }^{23}$, this implies that a planar thin film placed at a node will be perfectly transparent, while the same thin film will be strongly excited if placed at an anti-node. With respect to absorption, which is limited to $50 \%$ in planar materials illuminated by a travelling plane wave ${ }^{24}$, standing wave illumination allows absorption to be controlled from 0 to $100 \%$ in the ideal case $e^{7}$. Such performance in the optical part of the spectrum, as is relevant to optical fibre technology, may be approximated with materials that are thin compared to the optical wavelength and exhibit equal levels of transmission and reflection in addition to around 50\% travelling wave absorption. This combination of parameters may be achieved for example in nanostructured plasmonic metamaterials ${ }^{8}$ and 30layer graphene ${ }^{11,25}$.

The planar absorber used here is a plasmonic metamaterial consisting of a 70-nm-thick gold film perforated with an array of asymmetrically split ring apertures, as previously deployed in free-space demonstrations of coherent light absorption and transparency ${ }^{8}$. The dependence of this structure's transmission, reflection and absorption characteristics on aperture size and
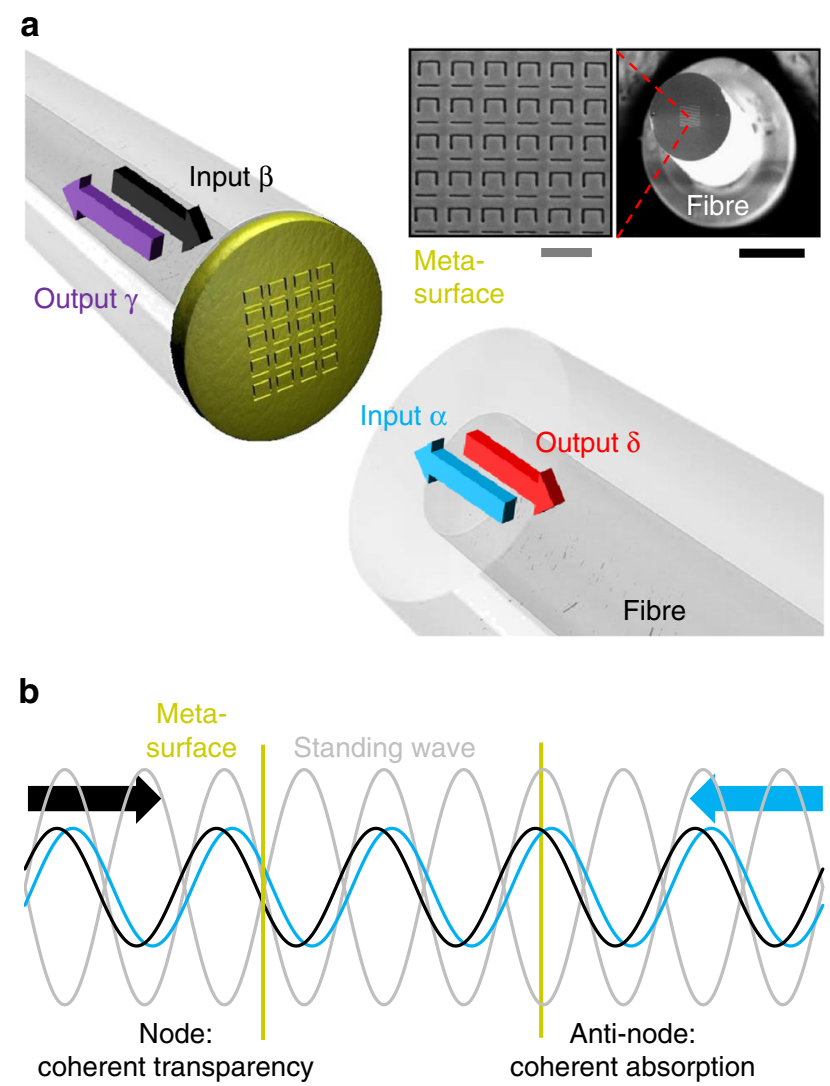

Fig. 1 Coherent interaction of light with light on a metasurface. a Coherent optical input signals $\alpha$ and $\beta$ interact on a metasurface absorber, generating output signals $\gamma$ and $\delta$. The metasurface has been fabricated by nanostructuring the central $25 \times 25 \mu \mathrm{m}^{2}$ of a 70 -nm-thick gold layer covering the cleaved end-face of a polarization-maintaining single-mode silica fibre (inset scanning electron microscope images, black scale bar 100 $\mu \mathrm{m}$, grey scale bar $1 \mu \mathrm{m})$. $\mathbf{b}$ The counterpropagating coherent input signals form a standing wave wherein the metasurface can be located at a position of destructive interference of electric fields (node) where absorption is suppressed or at a position of constructive interference (anti-node) where absorption is increased. In the ideal case, absorption can correspondingly be controlled from 0 to $100 \%$

geometry $^{26}$ is well understood, allowing for easy optimization throughout the optical telecommunications bands. Our switch operates at wavelengths around $\lambda=1550 \mathrm{~nm}$, where its $70 \mathrm{~nm}$ thickness corresponds to $\lambda / 22$. The metamaterial structure was fabricated by thermal evaporation of gold and subsequent focused ion beam milling on a $25 \times 25 \mu \mathrm{m}^{2}$ area covering the core of a cleaved polarization-maintaining single-mode silica fibre (see inset to Fig. 1a), with the symmetry axis of the metamaterial aligned to the slow axis of the fibre (see Methods section for details). The fibre output was coupled to a second cleaved polarization-maintaining optical fibre using two microcollimator lenses to realize an in-line fibre metadevice (Fig. 2a).

The metadevice is terminated with standard FC/APC fibre connectors and it was characterized in a fibre interferometer assembled from standard polarization-maintaining fibre components (Fig. 2a). The output of a fibre-coupled CW laser was split along two paths of similar length, with one path containing an electro-optical phase or intensity modulator and the other containing a variable attenuator to allow balancing of the power propagating along the two paths. The paths were then recombined within the metadevice and the output signals were 
a

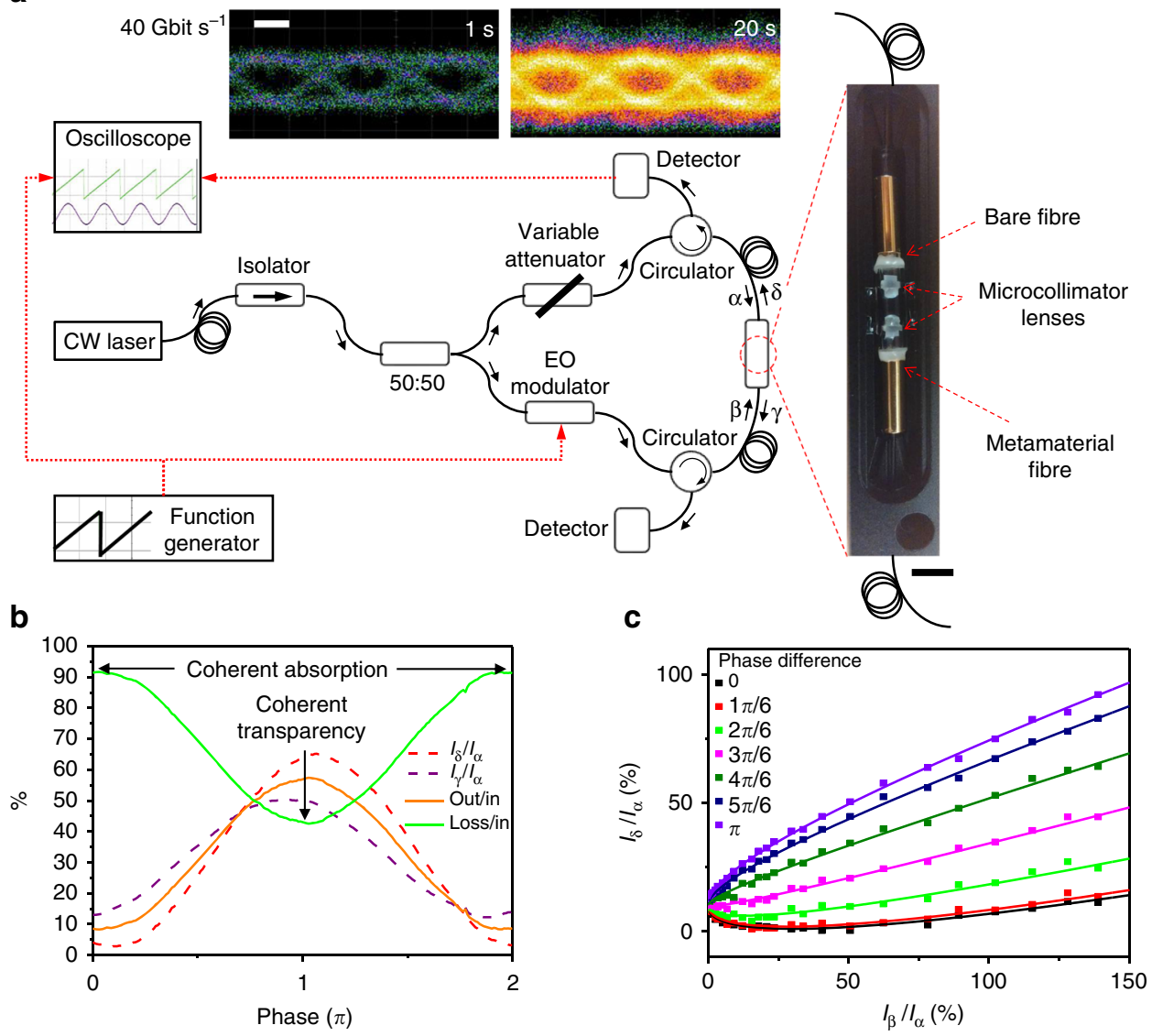

Fig. 2 The packaged metadevice and its properties. a Schematic representation of the fully fiberized experimental setup with a photograph of the packaged metadevice (without lid, black scale bar $5 \mathrm{~mm}$ ) consisting of the metasurface-covered fibre (Fig. 1a) coupled to a bare fibre end using a pair of microcollimator lenses. The inset shows eye diagrams of the intensity of output channel $\delta$ recorded for intensity modulation of input channel $\beta$ at $40 \mathrm{Gbit} \mathrm{s}^{-1}$, where colour indicates counts and the white scale bar indicates $10 \mathrm{ps}$. b Measured output intensities $I_{\gamma}$ and $I_{\delta}$ (relative to $I_{\alpha}$ ) as well as the total output power and metadevice losses (relative to the total input power) as a function of the phase difference between the input signals at the metasurface at a wavelength of $1550 \mathrm{~nm}$. c Measured output intensity $I_{\delta}$ (data points) relative to the fixed input intensity $I_{\alpha}$ as a function of input intensity $I_{\beta}$ for various phase differences between the input beams, with fits (lines), again at $1550 \mathrm{~nm}$

detected via circulators using an oscilloscope (see Methods section for a more detailed description). We note that practical applications would require some means of active stabilization of the optical path lengths, however the system shown here is sufficient to characterize the principle of operation of the fiberized switch. This is illustrated by the eye diagrams in Fig. 2a, which show that the eye closes on a timescale of seconds due to phase drift in the interferometer.

Figure $2 \mathrm{~b}$ shows the phase-dependent output intensities $I_{\gamma}$ and $I_{\delta}$ relative to the input intensity $I_{\alpha}=I_{\beta}$ as a function of the phase difference between the inputs at a wavelength of $1550 \mathrm{~nm}$. The overall output power can be controlled from about $9 \%$ to about $57 \%$ of the total input power, where a low output level corresponds to constructive interference of the incident waves on the metasurface and thus coherent absorption, while a high output level corresponds to coherent transparency. Both output signals display a similar phase-dependence; however, the phasedependent output intensity $I_{\delta}$ offers somewhat higher contrast and therefore we focus on this output. It should be noted that, for an ideal metadevice containing a perfectly symmetric ultrathin absorber showing 50\% single-beam absorption (with no other loss mechanisms) and equal reflection and transmission for both directions of propagation, both output intensities would be identical and modulated from complete absorption to perfect transmission. Differences between the measured output channels arise in the present case from the asymmetric construction of our metadevice that contains a metasurface fabricated on the glass/air interface of one of the optical fibres. The whole metadevice exhibits about $24 \%$ single-beam transmission, $18 \%(8 \%)$ reflection and $58 \%(68 \%)$ losses for a single input signal $\alpha(\beta)$. However, only part of these losses correspond to metasurface absorption that can be coherently controlled, while other sources of loss include the fibre connections of the metadevice, scattering and unwanted reflections within the microcollimator and fabrication imperfections such as imperfect alignment of the metasurface orientation with the slow axis of the fibres.

The nonlinear functionality of the switch is illustrated by Fig. $2 c$, which shows how the output intensity $I_{\delta}$ depends on the input intensity $I_{\beta}$ while $I_{\alpha}$ remains constant for various phase differences between the input beams. The measured output intensity $I_{\delta}$ as a function of input $I_{\beta}$ is nonlinear and generally follows the behaviour predicted by Fang et al. ${ }^{7}$. For input phase differences of less than $\pi / 2$ it is also nonmonotonic-counterintuitively the output intensity decreases with increasing input intensity and reaches a minimum before increasing when $I_{\beta}$ becomes large. For an input phase difference of $\pi / 2$, changes in the measured output intensity $I_{\delta}$ are approximately proportional to changes in the input intensity $I_{\beta}$. For larger input phase 
differences, $I_{\delta}$ flattens with increasing $I_{\beta}$, but is steep for small $I_{\beta}$ suggesting possible applications in small signal amplification. Thus, the results presented in Fig. 2b, $c$ show that large changes of the metadevice output result from modulation of phase or intensity of one of the metadevice inputs.

All-optical signal processing. All-optical signal processing operations with input/output relations analogous to logical functions may now be realized in the network by exploiting coherent transparency and/or coherent absorption in the metadevice. In what follows, binary logical states encoded in beams of equal intensity but opposite phase in the metasurface plane are denoted ' + ' and ' - ', while opposing states encoded as low/high intensity are denoted ' 0 '/' 1 '. Consider, in the first instance, the case of mutually coherent, binary, phase-modulated input signals + and - : constructive interference of identical bits $\alpha$ and $\beta$ will lead to coherent absorption on the metasurface, while destructive interference of opposing bits will lead to coherent transparency, producing an intensity-modulated (0/1) output $\alpha$ XOR $\beta$. The behaviour of an ideal switch is summarized in Table 1 and the measured behaviour of the experimental metadevice is shown in

\begin{tabular}{|c|c|c|c|}
\hline \multicolumn{2}{|c|}{ Input phase states } & \multicolumn{2}{|c|}{ Ideal output intensities $I_{\gamma}=I_{\delta}$} \\
\hline$\alpha$ & $\boldsymbol{\beta}$ & $\begin{array}{l}\theta_{\text {ext }}=0 \\
\alpha \text { XOR } \beta\end{array}$ & $\begin{array}{l}\theta_{\text {ext }}=\pi \\
\alpha \text { XNOR } \beta\end{array}$ \\
\hline+ & + & 0 & 1 \\
\hline+ & - & 1 & 0 \\
\hline- & + & 1 & 0 \\
\hline - & - & 0 & 1 \\
\hline
\end{tabular}

Fig. 3a, for a modulation frequency of $10 \mathrm{kHz}$. The switch clearly presents XOR functionality with high contrast $(>10 \times)$ between the output states. The intensity of the output logical 1 is about $30 \%$ lower than in the ideal case due to losses within the metadevice. Note that the XOR function can be inverted to $\alpha$ XNOR $\beta$ by providing an additional external phase-shift $\theta_{\text {ext }}=\pi$ to one of the input signals. Furthermore, a fixed input signal $\beta$ of + or could be used to map the phase-modulated signal $\alpha$ to an intensity-modulated signal with (NOT $\alpha$ ) or without (IDENTITY $\alpha)$ inversion.

The network can also perform signal processing operations analogous to logical functions on intensity-modulated input data. The simplest example is a NOT function. Such inversion of an intensity-modulated signal $\alpha$ is achieved by leaving input beam $\beta$ always on with its phase adjusted such that coherent absorption will occur for simultaneous illumination of the metasurface from both sides $\left(\theta_{\text {ext }}=0\right)$. Input pulses $\alpha($ logical 1$)$ will be coherently absorbed resulting in low output (logical 0 ). On the other hand, low input signals $\alpha$ (logical 0 ) will allow light from input $\beta$ to reach the outputs (logical 1). For an ideal metadevice, the expected output intensities are 0 and $25 \%$ of the input intensity, respectively; our experimental device achieves about 5 and $23 \%$ at a modulation frequency of $10 \mathrm{kHz}$, which is more than sufficient to distinguish the logical states (Fig. 3b).

While the NOT function was based on coherent absorption, an AND function between binary intensity-modulated signals can be realized by exploiting coherent transparency. In this case, a phase shift is applied to one input signal, such that simultaneous illumination of the metasurface from both sides leads to coherent transparency $\left(\theta_{\text {ext }}=\pi\right)$. For an ideal device, this would lead to $100 \%$ output intensity for interaction of two pulses on the metasurface and at least $4 \times$ lower output intensity for any other combination of input bits (Table 2). Experimentally we observe the AND function with more than $3 \times$ contrast between the logical output states at a modulation frequency of $10 \mathrm{kHz}$. In principle,
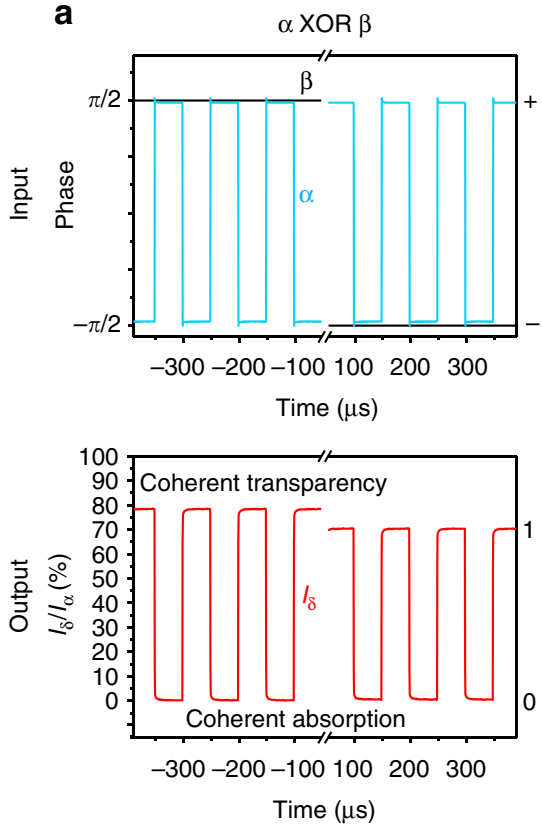

b
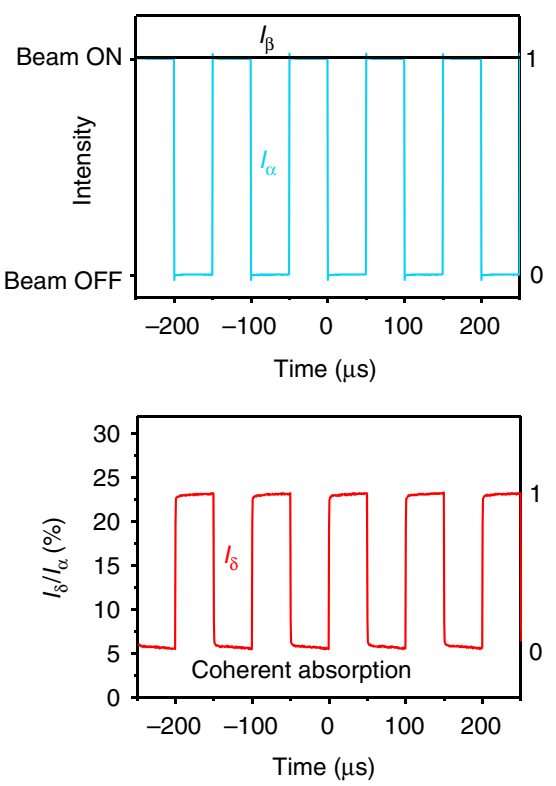
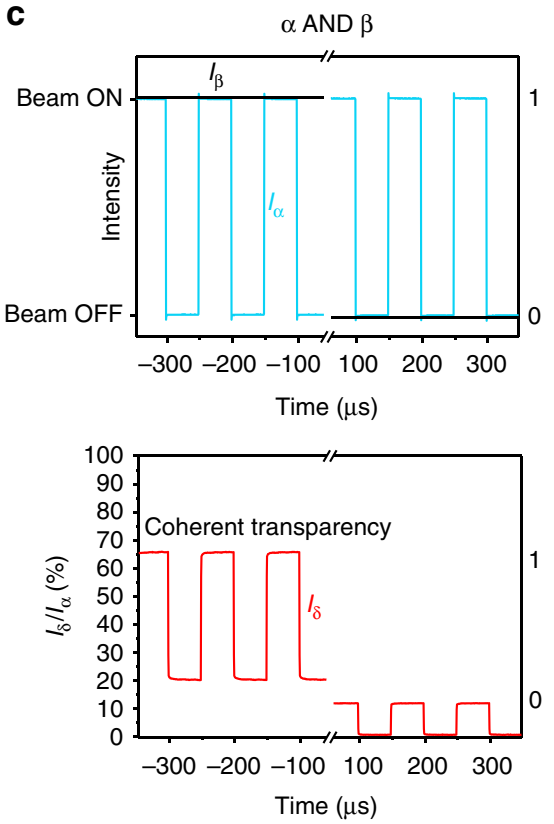

Fig. 3 All-optical signal processing at $10 \mathrm{kHz}$ at a wavelength of $1550 \mathrm{~nm}$. a XOR function between phase-modulated input signals $\alpha$ and $\beta$ producing an intensity-modulated output based on coherent absorption of identical bits and coherent transparency for opposing bits. $\mathbf{b}$ NOT function on a single intensity-modulated signal $\alpha$. The inversion of signal $\alpha$ in the presence of beam $\beta$ (which is always on) results from coherent absorption of incoming signal pulses when the metasurface is located at a standing wave anti-node. c AND function between intensity-modulated signals $\alpha$ and $\beta$ resulting from coherent transparency of the metasurface for simultaneous illumination from both sides when the metasurface is located at a standing wave node. The logical states are indicated on the right-hand side of each graph. Minor signal distortions are due to the limited bandwidth of the waveform generator 
other logical functions including XOR and OR for intensitymodulated signals can be realized for suitable choices of $\theta_{\text {ext }}^{22}$.

Gigabits per second and beyond. In practical systems, optical signals transmitted by optical fibres are modulated at $\mathrm{GHz}$ frequencies rather than $\mathrm{kHz}$, and also make use of a range of optical wavelengths. We therefore tested the metadevice at modulation frequencies 5-6 orders of magnitude higher than presented in Fig. 3 and at wavelengths ranging from 1530 to $1565 \mathrm{~nm}$ (telecommunications C-band), as illustrated in Figs. 4 and 5. To this end, the output of the fibre interferometer was amplified with an erbium-doped fibre amplifier (EDFA) which provided an average output power of $1 \mathrm{~mW}$. This conveniently ensures that the threshold power between logical 0 and logical 1 will always be close to $1 \mathrm{~mW}$. Figure $4 \mathrm{a}$ shows the XOR function on phasemodulated signals (as described above) now at a modulation frequency of $1.2 \mathrm{GHz}$, while Fig. $4 \mathrm{~b}$ shows the NOT function on an intensity-modulated signal also at $1.2 \mathrm{GHz}$. An AND function was realized by intensity modulation of the laser light entering the

\begin{tabular}{|c|c|c|}
\hline \multicolumn{2}{|c|}{ Input states } & \multirow{2}{*}{$\begin{array}{l}\text { Ideal output intensities } I_{\gamma}=I_{\delta} \\
\theta_{\text {ext }}=\pi \\
\alpha \text { AND } \beta\end{array}$} \\
\hline$\alpha=I_{\alpha}$ & $\beta=I_{\beta}$ & \\
\hline 1 & 1 & 1 \\
\hline 1 & 0 & 0.25 \\
\hline 0 & 1 & 0.25 \\
\hline 0 & 0 & 0 \\
\hline
\end{tabular}

interferometer (i.e. modulation before the 50:50 splitter shown in Fig. 2a) and the introduction of a path difference in the interferometer arms to delay the modulated signals $\alpha$ and $\beta$ relative to one another as illustrated by Fig. 4c. This is equivalent to an intensity-modulated bit sequence 0011 in channel $\alpha$ and 1001 in channel $\beta$, resulting in an output 0001, i.e. $\alpha$ AND $\beta$, in the detected output channel.

Figure 5 shows the NOT function of the switching network at a frequency of $10 \mathrm{GHz}$. The periodic input signal $\alpha$ was generated with a bit pattern generator and is equivalent to a bit sequence of 1011, which is inverted to become 0100 at a rate of $40 \mathrm{Gbit} \mathrm{s}^{-1}$. Measurements at wavelengths from 1530 to $1565 \mathrm{~nm}$ show successful signal inversion, thereby illustrating the broadband nature of the underlying coherent absorption effect across the full wavelength range of the tuneable laser used in the experiment. With $50 \mu \mathrm{W}$ peak power in each input channel and a 25 ps pulse duration per bit, the modulator's energy consumption is $2.5 \mathrm{fJ}$ per bit, which corresponds to about 20,000 photons per bit. Given that coherent absorption of single photons has been demonstrated $^{11}$, we expect that energy consumption in the attojoule per bit regime should be possible with a sufficiently sensitive detection system.

The modulation results obtained at $\mathrm{GHz}$ frequencies are similar to those obtained at $\mathrm{kHz}$ frequencies. At $\mathrm{GHz}$ frequencies, some distortion is apparent in both the signals used to drive the phase and intensity modulators as well as the measured output signal, where contrast is slightly reduced. These distortions arise from the frequency response and background noise of the modulators and amplifiers used in the experiments. While our experimental equipment does not allow us to test the performance of the modulator in the fibre environment beyond $40 \mathrm{Gbits}^{-1}$, we expect that the switching network with a
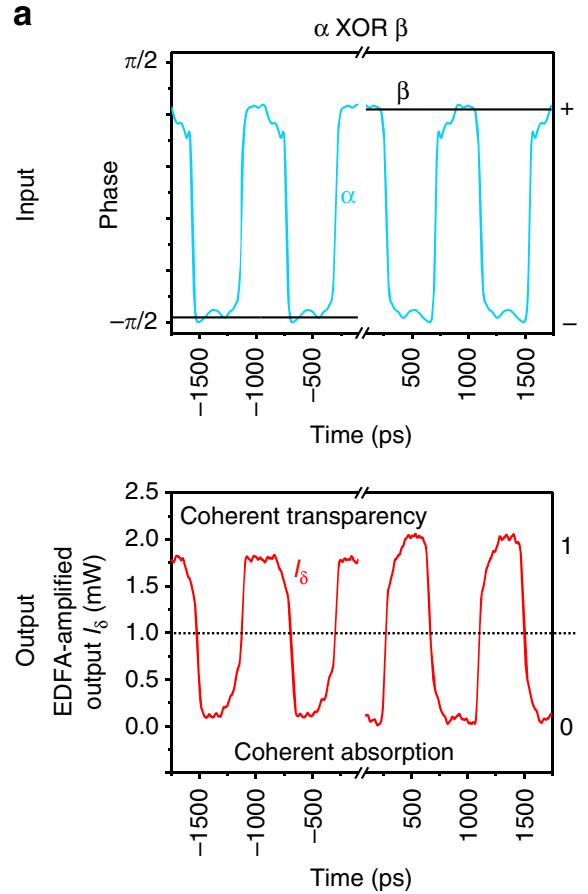

b
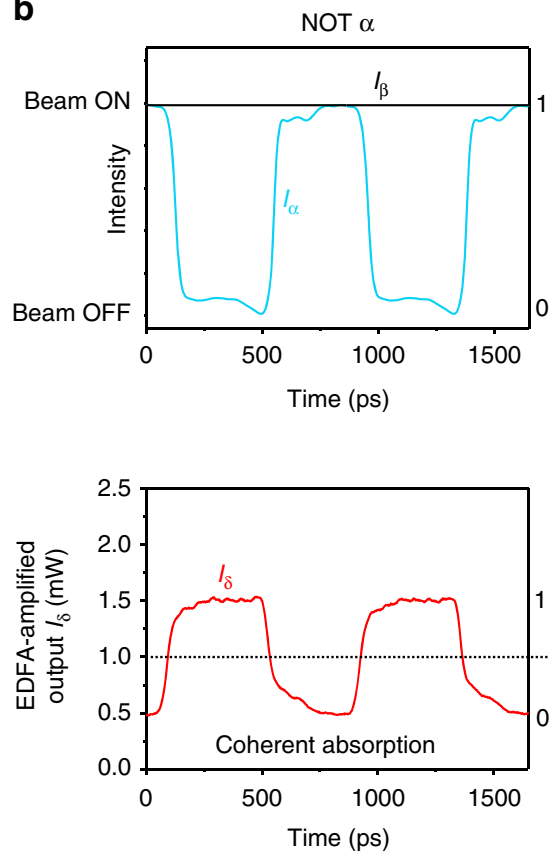
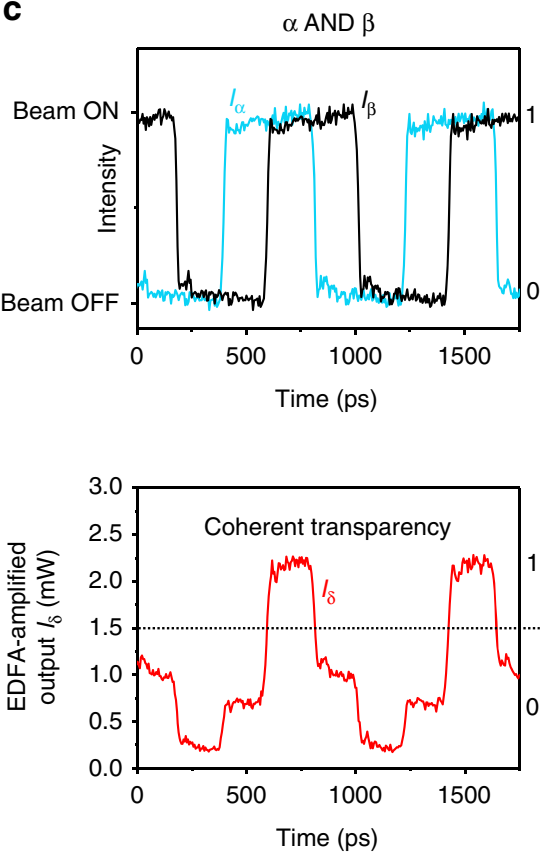

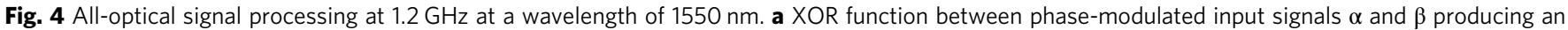
intensity-modulated output based on coherent absorption of identical bits and coherent transparency for opposing bits. $\mathbf{b}$ NOT function on a single intensity-modulated signal $\alpha$ in the presence of a constant beam $\beta$, resulting from coherent absorption of incoming signal pulses when the metasurface is located at a standing wave anti-node. c AND function on two intensity-modulated signals $\alpha$ and $\beta$ resulting from coherent transparency of the metasurface for simultaneous illumination from both sides when the metasurface is located at a standing wave node. The elevated noise level in $\mathbf{c}$, as compared to $\mathbf{a}$, $\mathbf{b}$, is due to a change in the experimental configuration (described in Methods). The logical states are indicated on the right-hand side of each graph 


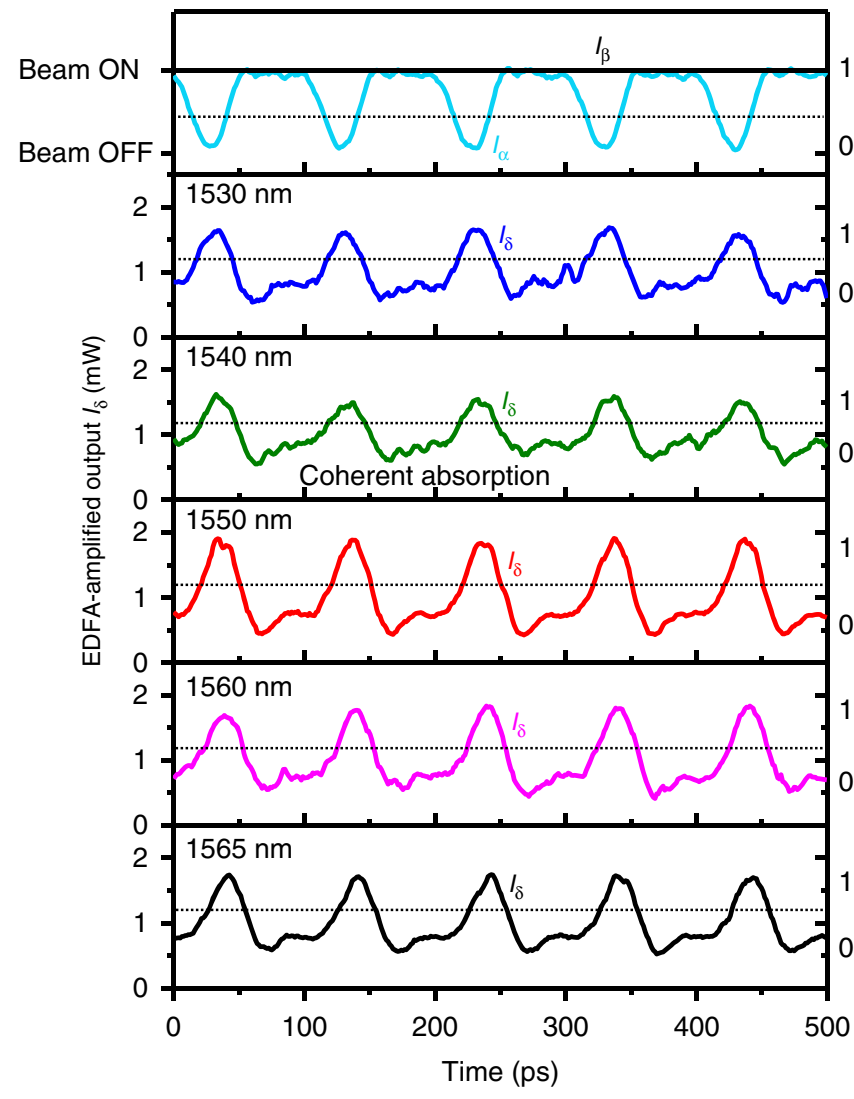

Fig. 5 Broadband inversion NOT $\alpha$ of a $40 \mathrm{Gbit} \mathrm{s}^{-1}$ input signal $\alpha$ at wavelengths from 1530 to $1565 \mathrm{~nm}$. The input signal corresponds to an intensity-modulated bit pattern 1011 repeating at $10 \mathrm{GHz}$ (top);

corresponding output traces for different wavelengths (below) show that the metadevice inverts the bit pattern in all cases. Beam $\beta$ is continuously in the on state (logical 1). The logical states 1 and 0 are indicated on the righthand side and separated by a horizontal dotted line on each graph

metamaterial absorber can in principle operate at much higher frequencies. Indeed, the underlying phenomena of coherent absorption and coherent transparency in plasmonic metamaterials occur on timescales as short as $10 \mathrm{fs}$, implying a potential bandwidth on the order of $100 \mathrm{THz}^{10}$. Based upon the spectral width of its plasmonic absorption peak, the metamaterial used in the present study may be expected to efficiently absorb pulses as short as $40 \mathrm{fs}$, corresponding to a potential bandwidth of tens of $\mathrm{THz}$ (Supplementary Note 1). However, such bandwidth will be difficult to realize in a fiberized switch due to dispersion limitations of the fibres.

\section{Discussion}

Even though we report a proof-of-principle demonstration, our results show that the coherent interaction of light with light on ultrathin films can be used to perform signal processing functions with high bandwidth and high contrast on signals carried by telecoms fibres. Improved device performance would result from a more symmetric design wherein the nanoscale thickness absorber is in contact with the same material on both sides, which may be achieved for instance by depositing a thin glass layer over the metasurface or via a bespoke splicing technique. The metamaterial design itself could also be improved to achieve $50 \%$ single-beam absorption and identical 25\% transmission and reflection characteristics from both sides as well as polarization independence. Another option may be to replace the metamaterial with multi-layer graphene $\mathrm{e}^{11,25}$.
All-optical signal processing applications based on coherently controlling the absorption of light with light promise extremely high bandwidth and extremely low energy requirements, but they will require mutually coherent signals and phase stability. Mutual coherence is most easily achieved in local systems, where multiple signals are derived from the same seed laser. Indeed, as locally coherent networks become part of the mainstream telecommunications agenda ${ }^{27-29}$, coherent all-optical data processing may become a realistic proposition, particularly in miniaturized integrated optics and silicon photonic chips ${ }^{30}$, where phase stability is more easily achieved than in large-scale fibre networks. In order to go beyond simple single-step logical functions, cascading of multiple coherent operations will also need to be explored. Such cascading is likely to require signal regeneration techniques as the XOR function converts phase-shift keying to amplitudeshift keying (though in principle without insertion loss and with unlimited contrast), while the AND function suffers from limited contrast of $6 \mathrm{~dB}$ (though in principle without insertion loss) and the NOT function suffers from insertion loss of $6 \mathrm{~dB}$ (in principle with unlimited contrast).

It is notable that metasurfaces can be engineered to enable a broad range of switching and control functions, which may be used to implement various signal processing functionalities ${ }^{7,22,31}$. Devices based on coherent perfect absorption (as considered here) and lossless devices are limiting cases among a much wider range of possibilities. Beyond all-optical data processing, potential applications of coherent metadevices include small signal amplification and coherence filtering ${ }^{8}$.

The switching network demonstrated here is one example among many opportunities arising from fibre-integration of metasurfaces, which could also be used to control, for example, focusing, polarization, spectral characteristics, propagation direction and angular momentum of light ${ }^{32-34}$.

In summary, we report a metamaterial-based fiberized switching network for all-optical signal processing that is compatible with optical telecommunications fibre components. The multi-functional switch can perform effectively-nonlinear signal processing functions including input/output relations analogous to XOR, AND and NOT operations, and the underlying mechanism of coherent transparency and coherent absorption is compatible with single-photon signals and $100 \mathrm{THz}$ bandwidth. We therefore anticipate that such fiberized metadevices may provide solutions for quantum information networks as well as orders-of-magnitude improvements in speed and energy consumption over existing nonlinear approaches to all-optical signal processing in coherent information networks.

\section{Methods}

Metadevice fabrication. For the realization of the metadevice, the metamaterial was fabricated on the cleaved end face of a polarization-maintaining single-mode fibre as described above. The metamaterial nanostructure was fabricated with its symmetry axis aligned with the slow axis of the Panda-style fibre. In order to allow metamaterial illumination from both sides, the metamaterial-covered fibre was interfaced with a second polarization-maintaining fibre using a microcollimator arrangement consisting of a pair of microlenses. Specifically, the metamaterialcovered fibre end was inserted into a fibre ferrule and positioned at the focal point of an anti-reflection-coated microlens to fabricate a compact microcollimator that was stabilized by a glass capillary shell. The second cleaved fibre tip was mounted in the same way. Using kinematic mounts, the two microcollimators were aligned with each other by maximizing optical coupling efficiency and polarization contrast. The aligned microcollimator arrangement was then fixed with UV-cured adhesive, stabilized with an outer glass capillary shell and placed in an anodized aluminum enclosure for additional protection (Fig. 2a).

Experimental metadevice characterization. Experimental characterization was performed using the interferometer arrangement presented in Fig. 2a. All fibre components were based on Panda-style polarization-maintaining single-mode fibres. In all cases the incident electric field was oriented parallel to the symmetry axis of the metamaterial nanostructure (slow axis of the fibre). Measurements at 
$\mathrm{kHz}$ frequencies used the $180 \mu \mathrm{W}$ output of a fibre-coupled $1550 \mathrm{~nm}$ CW laser diode and were recorded using InGaAs photodetectors and an oscilloscope (Agilent Technologies DSO7104A). They were calibrated by taking into account the insertion losses of fibre components, such that $I_{\alpha}, I_{\beta}, I_{\gamma}$ and $I_{\delta}$ in Figs. $2 \mathrm{~b}, \mathrm{c}$ and 3 correspond to the intensities entering and leaving the metadevice's fibre connectors. The peak input power at the metadevice input connector $I_{\alpha}=I_{\beta}$ was $10 \mu \mathrm{W}$. The input signal modulators used were low-loss electro-optical $10 \mathrm{Gbit} \mathrm{s}^{-1}$ phase and intensity modulators (EOspace) driven by a waveform generator (AM300 by Rohde \& Schwarz).

Measurements at $\mathrm{GHz}$ frequencies used a fibre-coupled tuneable $\mathrm{CW}$ laser (ID Photonics CoBrite-DX4). Here the EDFA-amplified output power was detected by an oscilloscope (Agilent Infiniium DCA-J 86100C) as described in the main text. The peak input power at the metadevice input connector $I_{\alpha}=I_{\beta}$ is $\sim 100 \mu \mathrm{W}$ in the case of Fig. $4 \mathrm{a}, \mathrm{b}, 30 \mu \mathrm{W}$ in Fig. $4 \mathrm{c}$ and $50 \mu \mathrm{W}$ in Fig. 5. The modulators used were low-loss electro-optical $10 \mathrm{Gbit} \mathrm{s}^{-1}$ phase and intensity modulators (EOspace) driven by an arbitrary waveform generator (Tektronix AWG7122C) and a radio frequency amplifier (LA Techniques) in the case of Fig. $4 \mathrm{a}, \mathrm{b}$, and a bit pattern generator (SHF $12100 \mathrm{~B}$ ) in the case of Figs. 2a (eye diagrams), 4c and 5.

Data availability. The data from this paper is available from the University of Southampton ePrints research repository: https://doi.org/10.5258/SOTON/D0172.

Received: 15 September 2017 Accepted: 30 November 2017 Published online: 12 January 2018

\section{References}

1. Singh, P., Tripathi, D. K., Jaiswal, S. \& Dixit, H. K. All-optical logic gates: designs, classification, and comparison. Adv. Opt. Technol. 2014, 275083 (2014).

2. Boyd, R. W. Nonlinear Optics, 3rd edn (Academic Press, MA, USA, 2008).

3. Almeida, V. R., Barrios, C. A., Panepucci, R. R. \& Lipson, M. All-optical control of light on a silicon chip. Nature 431, 1081-1084 (2004)

4. Xu, Q. \& Lipson, M. All-optical logic based on silicon micro-ring resonators Opt. Express 15, 924-929 (2007).

5. Nozaki, K. et al. Sub-femtojoule all-optical switching using a photonic-crystal nanocavity. Nat. Photonics 4, 477-483 (2010).

6. Willner, A. E., Khaleghi, S., Chitgarha, M. R. \& Yilmaz, O. F. All-optical signal processing. J. Lightwave Technol. 32, 660-680 (2014).

7. Fang, X., MacDonald, K. F. \& Zheludev, N. I. Controlling light with light using coherent metadevices: all-optical transistor, summator and invertor. Light Sci. Appl. 4, e292 (2015).

8. Zhang, J., MacDonald, K. F. \& Zheludev, N. I. Controlling light-with-light without nonlinearity. Light Sci. Appl. 1, e18 (2012).

9. Fang, X. et al. Ultrafast all-optical switching via coherent modulation of metamaterial absorption. Appl. Phys. Lett. 104, 141102 (2014).

10. Nalla, V., Valente, J., Sun, H. \& Zheludev, N. I. 11-fs dark pulses generated via coherent absorption in plasmonic metamaterial. Opt. Express 25, 22620-22625 (2017).

11. Roger, T. et al. Coherent perfect absorption in deeply subwavelength films in the single-photon regime. Nat. Commun. 6, 7031 (2015).

12. Pirruccio, G. \& Rivas, J. G. Modulated light absorption and emission of a luminescent layer by phase-controlled multiple beam illumination. Opt. Express 23, 18166-18180 (2015).

13. Pirruccio, G., Ramezani, M., Rodriguez, S. R. \& Rivas, J. G. Coherent control of the optical absorption in a plasmonic lattice coupled to a luminescent layer. Phys. Rev. Lett. 116, 103002 (2016).

14. Shi, J. et al. Coherent control of Snell's Law at metasurfaces. Opt. Express 22, 21051-21060 (2014)

15. Kita, S. et al. Coherent control of high efficiency metasurface beam deflectors with a back partial reflector. APL Photonics 2, 046104 (2017).

16. Rao, S. M. et al. Geometries for the coherent control of four-wave mixing in graphene multilayers. Sci. Rep. 5, 15399 (2015).

17. Mousavi, S. A., Plum, E., Shi, J. \& Zheludev, N. I. Coherent control of optical polarization effects in metamaterials. Sci. Rep. 5, 8977 (2015).

18. Roger, T. et al. Coherent absorption of N00N states. Phys. Rev. Lett. 117, 023601 (2016)

19. Altuzarra, C. et al. Coherent perfect absorption in metamaterials with entangled photons. ACS Photonics 4, 2124-2128 (2017).

20. Fang, X., Tseng, M. L., Tsai, D. P. \& Zheludev, N. I. Coherent excitation-selective spectroscopy of multipole resonances. Phys. Rev. Appl. 5, 014010 (2016).

21. Papaioannou, M., Plum, E., Valente, J., Rogers, E. T. \& Zheludev, N. I. Two-dimensional control of light with light on metasurfaces. Light. Sci. Appl. 5 , e16070 (2016).
22. Papaioannou, M., Plum, E., Valente, J., Rogers, E. T. F. \& Zheludev, N. I. Alloptical multichannel logic based on coherent perfect absorption in a plasmonic metamaterial. APL Photonics 1, 090801 (2016).

23. Plum, E. \& Zheludev, N. I. in Structured Surfaces as Optical Metamaterials Ch 4, 94-157 (Cambridge University Press, Cambridge, UK, 2011).

24. Thongrattanasiri, S., Koppens, F. H. L. \& García de Abajo, F. J. Complete optical absorption in periodically patterned graphene. Phys. Rev. Lett. 108, 047401 (2012).

25. Rao, S. M., Heitz, J. J. F., Roger, T., Westerberg, N. \& Faccio, D. Coherent control of light interaction with graphene. Opt. Lett. 39, 5345-5374 (2014).

26. Plum, E. et al. A combinatorial approach to metamaterials discovery. J. Opt. 13, 055102 (2011)

27. Kikuchi, K. in High Spectral Density Optical Communication Technologies. Optical and Fiber Communications Reports Vol. 6, Ch. 2, 11-49 (Springer-Verlag, Berlin/Heidelberg, 2010).

28. Slavík, R. et al. All-optical phase and amplitude regenerator for next-generation telecommunications systems. Nat. Photonics 4, 690-695 (2010).

29. Crivelli, D. E. et al. Architecture of a single-chip $50 \mathrm{~Gb} / \mathrm{s} \mathrm{dp-qpsk/bpsk}$ transceiver with electronic dispersion compensation for coherent optical channels. IEEE Trans. Circuits Syst. I, Reg. Papers 61, 1012-1025 (2014).

30. Dong, P. et al. Monolithic silicon photonic integrated circuits for compact $100^{+}$ $\mathrm{Gb} / \mathrm{s}$ coherent optical receivers and transmitters. IEEE J. Sel. Top. Quant. Elect. 20, 150-157 (2014)

31. Plum, E., MacDonald, K. F., Fang, X., Faccio, D. \& Zheludev, N. I. Controlling the optical response of $2 \mathrm{~d}$ matter in standing waves. ACS Photonics, Article ASAP, https://doi.org/10.1021/acsphotonics.7b00921 (2017).

32. Zeng, J. et al. Manipulating complex light with metamaterials. Sci. Rep. 3, 2826 (2013).

33. Yu, N. \& Capasso, F. Optical metasurfaces and prospect of their applications including fiber optics. J. Lightwave Technol. 33, 2344-2358 (2015).

34. Principe, M. et al. Optical fiber meta-tips. Light Sci. Appl. 6, e16226 (2017).

\section{Acknowledgements}

We thank Daniele Faccio, Jun-Yu Ou and Vassili Savinov for advice and fruitful discussions. This work is supported by the UK's Engineering and Physical Sciences Research Council (grant EP/M009122/1) and the MOE Singapore (grant MOE2011-T3-1-005).

\section{Author contributions}

A.X. fabricated the metadevice and characterized it at $\mathrm{kHz}$ frequencies. Y.J. and A.X packaged the metadevice. I.D., A.X., E.P. and C.L. conducted the GHz frequency experiments. V.N. conducted the supplementary ultrafast experiments. E.P., A.X. and N.I.Z. wrote the paper. All authors discussed the results and read the manuscript. E.P., K.F.M., P.P., D.J.R. and N.I.Z. supervised the work.

\section{Additional information}

Supplementary Information accompanies this paper at https://doi.org/10.1038/s41467 017-02434-y.

Competing interests: The authors declare no competing financial interests.

Reprints and permission information is available online at http://npg.nature.com/ reprintsandpermissions/

Publisher's note: Springer Nature remains neutral with regard to jurisdictional claims in published maps and institutional affiliations.

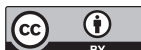

Open Access This article is licensed under a Creative Commons Attribution 4.0 International License, which permits use, sharing, adaptation, distribution and reproduction in any medium or format, as long as you give appropriate credit to the original author(s) and the source, provide a link to the Creative Commons license, and indicate if changes were made. The images or other third party material in this article are included in the article's Creative Commons license, unless indicated otherwise in a credit line to the material. If material is not included in the article's Creative Commons license and your intended use is not permitted by statutory regulation or exceeds the permitted use, you will need to obtain permission directly from the copyright holder. To view a copy of this license, visit http://creativecommons.org/ licenses/by/4.0/.

(c) The Author(s) 2018 